Conclusions Improving community safety is complex and the collaboration necessary to address injury prevention is challenging but not impossible. The PPSCN is an essential component to developing the capacity of communities to focus on the adoption of an integrated approach to planning and delivery based on the available evidence. From the collective experience, the benefits of having all sectors working together in a coordinated and collaborative way, forming partnerships to promote safety, manage risk and develop safer environments leads to an increase in the overall safety of members.

\section{DOES ONE SAFE CITY MODEL FIT EVERY COMMUNITY? EVALUATION OF 'BETEREM IN THE CITY' IN ISRAEL}

${ }^{1}$ Sharon Levi, ${ }^{2}$ Yuval Paldi, ${ }^{1}$ Michal Bar Doron, ${ }^{1}$ Orly Silbinger. ${ }^{1}$ Beterem-Safe Kids Israel; ${ }^{2}$ Tovanot- Organisational Evaluation, Planning and Consulting, Israel

10.1136/injuryprev-2016-042156.320

Background 'Beterem in the City' is a model developed in 2008, based on criteria established by Safe Communities, to promote and manage child safety in municipalities. The model incorporates components of public health, organisational consultation and safety management and is tailored to unique characteristics of municipalities in Israel. A formative and summative evaluation of program effectiveness was conducted.

Methods The three year evaluation process comprised various methods. Quantitative tools included: 1. Surveys with program directors and managers in 23 cities 2. Surveys with organisational consultants; 2. Surveys of safe behaviour in four cities. Qualitative tools included: 1 . Interviews with program developers and stakeholders; 2. In-depth review of program implementation in four cities, based on interviews and program documentation

Results The evaluation indicates that the implementation of the model is incomplete and demands improvement. Those components that are implemented in full demand less time, resources, and expertise as compared to the components that were not implemented. Program components that were found to be correlated with positive outcomes include effective management and utilisation of the organisational consultation hours.

While 'Beterem in the City' has potential to lead organisational change and increase child safety over time, the current model is ambitious and may not be suitable for the organisational culture and management in most municipalities in Israel. In spite of these results, the evaluation points to success in implementing the model and better outcomes over time and in cities with stronger and more evolved management structures.

Conclusions 'Beterem in the City' needs to be adapted to different types of municipalities in Israel, including Arab cities and cities with a low socio-economic population. In order to implement the model effectively additional resources, from national bodies, will need to be directed to the program.

\section{DEVELOPING THE ESTABLISHMENT OF "COMMUNITY CHECKPOINTS TO REDUCE ROAD TRAFFIC ACCIDENTS" IN KHONKAEN PROVINCE}

\footnotetext{
${ }^{1}$ Patcharawadee Pirunsuntorn, ${ }^{2}$ Wilai Thanalad, ${ }^{1}$ Adisak Khongwattananont, ${ }^{1}$ Sompong Jaroongjittanusonti, ${ }^{3}$ Anuchar Sethasathien. ' Khonkaen Provincial Public Health Office; ${ }^{2}$ Kranuan Crown Prince Hospital; ${ }^{3}$ National Institute for Emergency Medicine
}

Background As a result of the socio-economic changes, the traffics and the transportations have been increasing. According to the projection by Khonkaen Province Public Health Office, there is higher tendency for the inappropriate road traffic behaviour and the death rate during 2015-2019 and it is three times lower than the actual recorded figures. This can result in the higher costs for medical treatments for over 147 million baht a year. The accidents and the large portion of the costs are mainly due to the use of motorcycles on the roads in communities and villages.

Description of the problem The government; therefore, has officially announced its policy of "Decade of Action for Road Safety 2011-2020" as the national agenda responsible by five major government units who have run their actions in solving the problems of traffic accidents consecutively. Several projects successfully served the units' roles and responsibilities. However, despite the success and the same objective, there has been no integration of the work procedures among the networks of those units. Khonkaen provincial governor; therefore, has issued a policy to have a safety traffic measure by establishing the "community checkpoints to reduce road traffic accidents" with the objective to promote the development of a measure to prevent the road traffic injuries in the communities of all districts.

Results The results showed that, for all the 26 districts, the network leaders of each district had been promoted for higher potential. Based on the local context, district committees were formed with road safety action plan for community checkpoint operation to reduce traffic accidents. The real-time information for supporting the work operation as required by each unit in each area was processed systematically, accurately, and completely. The survey for the fundamental information, at-risk points, vehicles, environments, roads, and warning signs was conducted with welfare and financial support from the local governments and the organisations from private sector who participating in the project. All the parties concerned felt encouraged, valuable, and proud of the results. As evaluated through the one-page summary and the lesson learned from the community checkpoint operation, the project created a social tendency for realisation alert of safety road traffic behaviours which was obviously enhanced widely among the networks of all levels with the understanding of the integration of the work procedures in solving the road traffic accidents. The success of each district was found depending on the leaders' potential, the work cooperation, the team work communication, details of work procedures, and the experience of each team.

Conclusions The development of establishing the "Community Checkpoints to Reduce Road Traffic Accidents" in Khonkaen Province was found successful with support from the network leaders from each district, strong communities, and the participations of all organisations which helped reducing the road traffic accidents. The community checkpoints should be conducted with understanding, clear description of the working roles and details such as instruction for appointments and the legal protection for all the parties concerned should also be provided. The successful lesson should be enhanced and used as the model of the province and applied with other districts for creating strong and sustainable checkpoints in each community. 


\section{WORKING FROM THE INSIDE OUT: A CASE STUDY OF MACKAY SAFE COMMUNITY}

${ }^{1}$ Dale Hanson, ${ }^{2}$ Colleen Gunning, ${ }^{3} J u d y$ Rose, ${ }^{1}$ Kathryn McFarlane, 'Richard Franklin. ${ }^{1}$ James Cook University, Australia; ${ }^{2}$ Central Queensland University, Australia; ${ }^{3}$ Mackay Hospital and Health Service, Australia

10.1136/injuryprev-2016-042156.322

Background Mackay Safe Community (MSC) was established in 2000 in response to high injury rates in the region. A community-based intervention using the International Safe Communities (ISC) model was considered strategic.

The ISC program advocates a systematic, all injury, all age group, all situation, community-based approach to injury prevention and safety promotion. MSC assumed an ecological perspective, incorporating targeted safety promotion campaigns reinforced by supportive environments and policy. By involving the community in finding its own solutions, MSC attempted to catalyse structural, social and political changes that empowered the community and ultimately, individuals within the community, to modify their environment and their behaviour to reduce the risk of injury.

Method This study used Social Network Analysis to analyse the social resources mobilised by the network. Using a snowballing methodology, the chain of relationships that constitute Mackay Safe Communities and its support network was elucidated.

Results A community network consisting of 118 members and an external support network of 50 members was established. A social network analysis conducted in 2004 indicated that the network doubled its cohesiveness while simultaneously doubling the bridging and linking relationships necessary to mobilise the resources required to implement its safety promotion agenda. A $12 \%$ reduction in Emergency Department injury presentations to Mackay Base Hospital was observed over the four-year period from 2000 to 2004. Mackay Safe Community became the $81^{\text {st }}$ International Safe Community on the $31^{\text {st }}$ August 2004.

Conclusions MSC can only be understood in its ecological context. While it was rich in social resources, human and financial resources were largely controlled by external agencies. The productivity of MSC was vulnerable to the changing policy priorities of external sponsoring agents and critically dependent on the advocacy skills of its leaders.

\section{SAFETY PROMOTION AND INJURY PREVENTION LANDSCAPE FOR CHILDREN AND THEIR FAMILIES - SAFE COMMUNITIES IN AUSTRALIA}

Barbara Minuzzo. Co-Chair Australian Safe Communities Foundation (ASCF) and Victorian Safe Communities Network (VSCN) Secretariat

\subsection{6/injuryprev-2016-042156.323}

Background Australia has national (Australian Injury Prevention Network (AIPN)) and state (Victorian Safe Communities Network (VSCN)) level networks working together. Each network was established as an independent membership based incorporated association. (The two networks gave community safety professionals a forum for support and learning when Victoria's first two municipalities were designated as International Safe Communities in 1996). The role of these networks is to support government, business and the community to develop and promote safety strategies designed to minimise the impact of unintentional injury, crime, violence and emergency situations. More recently, the Australian Safe Communities Foundation (ASCF) was established.

Methods The networks cover the nation - all attempts are made to ensure representation from each state and territory occurs at election time for both the Australian Safe Communities Foundation board and the Australian Injury Prevention Network. An eight member elected Executive Committee supported by a Secretariat manages the Victorian Safe Communities Network. Ideally there is representation from the diverse sectors working in community safety including practitioners, researchers, and policymakers.

Dissemination of information from all three organisations to its members has evolved over time from a newsletter and online listserver for members - to a Blog, Twitter, and E-Bulletin news. This approach recognises that the networks need to change how they operate to respond to the needs of the community.

Activities undertaken have also evolved over time from consultations/support/certification as a safe community, face-to-face workshops/seminars, and a yearly conference to a conference every two years, online webinars, some face to face seminars, consultations with its members and writing position papers to influence Government.

Sustainability of the three organisations and the success of safe communities already long established was borne of the strong commitment by individuals and a shift in culture, and policy at key stakeholder and local government and organisational level. This culture shift encourages partnerships, networks and relationship building across sectors for the betterment of Safe Communities, by directing or participating in working groups. This provides a platform to progress safety issues locally as well as through government decision making processes. A healthy financially sustainable plan for the three networks continues to be an ongoing challenge depending on the national and state government agenda at the time

Results These networks facilitate a collaboration, to provide information and expertise on a range of community safety issues - across government and non-government agencies which influence policy development, support knowledge sharing and development and dissemination of resources.

These networks contribute to the expansion of safer communities, providing a platform for researchers and injury prevention practitioners to work with communities, implementing a broad multi-disciplinary approach to provide the best advice for individual community need. Tangible examples of the benefits of these networks include:

- Connecting communities seeking formal accreditation with other communities who have experience of the processes involved.

- Providing opportunities for networking and professional development through seminars and conferences conducted in partnership with other agencies and groups.

Conclusions Success comes to Australian local governments working to gain international safe community's accreditation The activities focus on promoting evidence based best practice and strength based approaches to early intervention. 